\section{M12 THE UTILISATION OF HELIOX21 IN A TERTIARY VOCAL CORD DYSFUNCTION SERVICE}

${ }^{1} \mathrm{~J}$ Haines, ${ }^{1} \mathrm{~A}$ Vyas, ${ }^{1} \mathrm{C}$ Slinger, ${ }^{2} \mathrm{~S} J$ Fowler. 'Lancashire Teaching Hospitals NHS Foundation Trust, Preston, UK; ${ }^{2}$ Institute of Inflammation and Repair, University of Manchester, Manchester, UK

\subsection{6/thoraxjnl-2015-207770.439}

Introduction Heliox 21 reduces the work of breathing in patients with extra-thoracic airway obstruction, is not curative or intended to replace other treatments. In our specialist service we have significant numbers of patients whom have history of regular hospitalisations, relating to poorly controlled vocal cord dysfunction (VCD). The gold standard for treatment is respiratory speech and language therapy (rSLT). We increasingly value Heliox 21 as an early adjunct to rSLT in severe patients who are establishing management strategies.

Aims To assess the impact of Heliox 21 on patient admission rate and self-reported experience, for patients with severe VCD.

Methods We retrospectively reviewed the rSLT caseload from June-December 2014. All patients with endoscopically confirmed VCD, greater than five VCD related hospitalisations prior to the commencement of VCD treatment and who were prescribed Heliox 21 for use in the community were included. We requested hospital admission data (from patient's GP and secondary care physicians) between June 2013-June 2015, and reviewed medical and rSLT notes for demographic information/ co-morbidity data/opinions of Heliox 21.

Results Five patients met the inclusion criteria, three were available for analysis; one male and two female (aged 23,43,57 years). All had treated co-morbidities of asthma (BTS step 5) and reflux. One patient had treated nasal disease. Six-months prior to community Heliox 21 administration the mean (range) number of hospital admissions was 11 (8-13); after instigation, during the same follow-up period, this reduced by $81 \%$ ( 2 admissions) and two patients had no hospitalisations. In all patients rSLT occurred simultaneously. Patient opinions included, 'heliox gives me time to start my therapy and means I don't ring 999 straight away,' and, 'heliox stops me from going to A\&E all the time.' Two patients, who had completed rSLT, had Heliox 21 removed as it was no longer needed.

Conclusions Heliox 21 has a positive impact on reducing VCD hospital admissions and is a low cost short-term solution ( $£ 160$ set-up, $£ 8.50$ month). This retrospective review has limitations; the impact of rSLT alone on admission rates needs to be compared. Further investigation is needed to examine the worth of Heliox 21 as an initial adjunct to rSLT, with consideration of how to prevent reliance.

\section{M13 CLINICAL CHARACTERISTICS AND MANAGEMENT OF PATIENTS PRESENTING TO THE "AIRWAYS CLINIC"; A SPECIALISED TERTIARY MULTI-DISCIPLINARY RESPIRATORY SERVICE}

1J Haines, ${ }^{1} \mathrm{~A}$ Vyas, ${ }^{1} \mathrm{C}$ Slinger, ${ }^{1} \mathrm{~N}$ Cheyne, ${ }^{2} \mathrm{~S} J$ Fowler. ${ }^{1}$ Lancashire Teaching Hospitals NHS Foundation Trust, Preston, UK; ${ }^{2}$ Institute of Inflammation and Repair, University of Manchester, Manchester, UK

\subsection{6/thoraxjnl-2015-207770.440}

Introduction Our specialist service manages patients with vocal cord dysfunction (VCD), chronic cough (CC) and dysfunctional breathing (DB), and referrals have grown exponentially since we introduced this novel multi-disciplinary (MDT) model in 2006.
The team comprises two severe asthma specialist physicians, two respiratory speech and language therapists (rSLT), a severe asthma nurse specialist, respiratory physiotherapist and clinical psychologist.

Aims To describe the clinical characteristics of those referred and assess utilisation of the multi-disciplinary structure.

Methods Patient demographics and clinical data were retrospectively collated from clinical records of patients referred between January and December 2014.

Results The service received 249 referrals. Excluding patients still in treatment or who failed to attend initial assessment, 141 complete data sets were available for analysis: $71 \%$ female; mean (range) age 55 (18-79) years. Assessment requests were for VCD (71\%), CC $(28 \%)$ or both $(1 \%)$ and over half were from NW severe asthma centres or extra-regional specialist centres. The majority of referrals were from hospital consultants (72\%), with the remainder from GPs (17\%) and AHPs (11\%). For VCD there was $73 \%$ agreement between the clinical suspicion on referral and nasendoscopic assessment. Approximately half had evidence of co-existent reflux $(52 \%)$ and a third (29\%) had nasal disease.

The majority were seen by more than one member of the MDT team; all by a specialist physician and a rSLT, $43 \%$ by respiratory physiotherapy, and 7\% clinical psychology.

In the VCD cohort $64 \%$ had a previous asthma diagnosis and this was confirmed in the majority (93\%) - 49(82\%) were $\geq$ Step 3 on BTS/SIGN guidelines; $43 \%$ were additionally referred for DB assessment; 78 flow volume loops were available and 31 $(40 \%)$ were suggestive of extra thoracic inspiratory airway obstruction.

Sixty-seven patients (48\%) received rSLT management [median (range) 4(1-8) sessions] with a further 16(11\%) scheduled to receive it post medical intervention. Of those who completed treatment, $63 \%$ had clinically improved presentation on discharge nasendoscopy.

Conclusions A large proportion of patients referred to a specialist service for patients with complex breathlessness require multi-disciplinary intervention. There is a significant incidence of VCD and DB in patients with severe complex asthma. Of those receiving rSLT interventions, outcome was extremely effective at reducing symptoms.

\section{Improving quality of care in COPD}

\section{M14 SURGICAL INTERVENTIONS FOR EMPHYSEMA: THE EXPERIENCE OF A COMMUNITY BASED COPD SERVICE}

${ }^{1} \mathrm{AB}$ Hardy, ${ }^{2} \mathrm{~S}$ Cowdell, ${ }^{2} \mathrm{P}$ Griffiths. ${ }^{1}$ Calderdale and Huddersfield NHS Foundation Trust, Halifax, West Yorkshire; ${ }^{2}$ Locala Community Services, Wakefield, West Yorkshire

\subsection{6/thoraxjnl-2015-207770.441}

Methodology Surgical interventions for emphysema have become more accessible in the last few years. ${ }^{1}$ We have reviewed the referral rate and outcomes from our community based COPD service to a tertiary surgical centre. Our COPD service includes a respiratory consultant and nurse specialist and has access to secondary care respiratory investigations such as HRCT and pulmonary function testing.

Results During the period April 2013-March 2015202 new referrals were made to the consultant led clinic of which 181 were subsequently diagnosed with COPD. 10 referrals for 